\title{
Secure, Autonomous, Intelligent Controller for Integrating Distributed Emergency Response Satellite Operations
}

\author{
William D. Ivancic \\ william.d.ivancic@nasa.gov \\ Phillip E. Paulsen \\ philli.e.paulsen@nasa.gov \\ NASA Glenn Research Center
}

\author{
Eric M. Miller \\ eric.miller@gd-ais.com \\ Steven P. Sage \\ Steven.Sage@gd-ais.com \\ General Dynamics - Advanced Information \\ Systems
}

\begin{abstract}
This report describes a Secure, Autonomous, and Intelligent Controller for Integrating Distributed Emergency Response Satellite Operations. It includes a description of current improvements to existing Virtual Mission Operations Center technology being used by US Department of Defense and originally developed under NASA funding. The report also highlights a technology demonstration performed in partnership with the United States Geological Service for Earth Resources Observation and Science using DigitalGlobe ${ }^{\circledR}$ satellites to obtain space-based sensor data.
\end{abstract}

\section{TABLE OF CONTENTS}

1. Objective 1

2. Background

3. VMOC Details 1

5. Current Concept of Operations

6. Proposed Future Enhancements

7. Disaster Relief Operational CONOPS …........................ 8

8. NASA's Real Time Mission Monitor ................................ 9

9. Technology Readiness Level Assessment ....................... 9

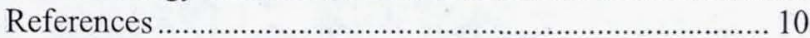

Appendix A - Acronyms .............................................. 10

\section{OBJective}

The United States Geological Service (USGS) Center for Earth Resources Observation and Science (EROS) Hazards Data Distribution System (HDDS) aims at providing a unified system of space data acquisition and delivery to those affected by natural or man-made disasters. The HDDS stores and provides dissemination access to a host of satellite and aerial data provider assets. Operations of these assets, critical to the success of disaster relief efforts worldwide, can be hampered by the lack of systems interoperability and automation, driving up the cost of providing services and interfering with the ability of the HDDS to provide timely data products. In conjunction with multiple government agencies and the United States Department of Defense (DOD), this task was aimed at applying the technology and lessons learned from multiple
NASA Earth Science Technology Office (ESTO) Advanced Information Systems Technology (AIST) development tasks [1-3]. The goal was to automate the coordination and acquisition of Earth observing resource data from a wide variety of sources (potentially national, international, commercial, NASA, and DOD). Lessons learned from this task and associated demonstrations have been provided to the International Charter of Space and Major Disasters and the general emergency response remote sensing community. Additionally, the newly created "web-based service" may be considered for a part of the larger Working Group on Information Systems and Services (WGISS) architecture.

\section{BACKGROUND}

NASA GRC pioneered the initial Virtual Mission Operations Center (VMOC) concept. Initial concepts were first demonstrated in November 2000 at NASA Johnson Space Center's (JSC) Inspection 2000 which allowed the public, industry, and other NASA organizations to view NASA technologies that were being or could be applied to Earthbound applications. Here, GRC, Goddard Space Flight Center (GSFC), JSC, and Veridian demonstrated secure, virtual, end-to-end mission operations over the Internet and NASA's Tracking and Data Relay Satellite System (TDRSS).

Working closely with General Dynamics - Advanced Information System (GD-AIS) (which acquired Veridian), Surrey Satellite Technology, the USA Space and Missile Defense Battle Lab, Universal Space Network (USN), the 14th Air Force, Joint Space Operations Center (JSpOC), Air Force Information Warfare Center, and numerous other companies, VMOC was developed, tested, and real-time commanding of a space asset was demonstrated in June of 2004. Included as part of this demonstration was the first secure, survivable, virtual mission operations and commanding of an element in space and the first secure, mobile retrieval of data from a sophisticated space-based system by remote, mobile users. The new technologies 
allowed non-space professionals to indirectly perform command and control of satellite assets.

ESTO's Advanced Technology Insertion (ATI) program provided funding to enhance the VMOC via the Secure Autonomous Automated Scheduling (SAAS) project. The SAAS objective was to monitor the United States Geological Survey (USGS) earthquake notification system, and have the General Dynamics (GD) developed VMOC located at NASA's Glenn Research Center (GRC) automatically schedule an image acquisition of the target area using via the Surrey Satellite Technology Ltd (SSTL), United Kingdom Disaster Monitoring Constellation-1 (UKDMC-1) satellite and download the acquired image via USN's ground terminals. The goal was to reduce the time between notification and availability of the image by using multiple ground stations with both schedule upload and payload download capability as well as demonstrate the ability to perform secure autonomous automated scheduling of on-orbit assets, triggered by available sensor webs (Tip and Cue). The UK-DMC's availability was obtained via machine-to-machine communications using SSTL's mission planning system. Access to/from the UK-DMC was via SSTL's and Universal Space Networks' (USN) ground assets. The availability and scheduling of USN's assets was also performed autonomously via machine-to-machine communications. All communication was via open Internet standards both on the ground and between ground and space. Although machine-to-machine scheduling was performed, this was performed using a mirror of SSTL's operational Mission Planning System. Full autonomous operation for VMOC to SSTL scheduler (MPS) to actual commanding of the space asset was not achieved due to funding limitations. SSTL required additional funding to add such automation into their mission planning system (machine-to-machine remote request to autonomous data delivery).

VMOC is currently the sole tasking tool for two DoD satellites (TacSat4 and ORS-1) and is being evaluated for use by the USGS for data generation in response to disasters and NGA for acquisition of commercial satellite imagery in support of the DoD.

\section{VMOC DETAILS}

For purposes of this task, earlier generation VMOC hardware and software housed at NASA Glenn Research Center (GRC) were upgraded with the appropriate software licenses and tools to provide a stable, secure, virtual front end for mission services, automated operations, data dissemination, and visualization. Additionally, key Advanced Information System Technology (AIST) funded Real Time Mission Monitor (RTMM) and technologies provided by the other DoD and EROS team members were also considered for introduction to the integrated system to allow new operations concepts to be developed and quickly fielded. Satellite services were automated and demonstrated using the integrated tool set.
Some of the detailed items that were added to the existing VMOC include:

- Ad hoc tasking generation

- Collection requirement management

- Rules based recurring tasking to meet higher level objectives

- Coordinated constellation-level scheduling of multiple space assets

- Use of live and simulated missions within the constellation

- Dynamic real-time modification to the constellation schedule based on real-time events

- Account management of assets

- Management of collections and data products on an asset by asset basis

- Human-in-the-loop release of requests to data providers

- Machine-to-machine interfaces for space-based sensor assets

- Interface support to DigitalGlobe

- Automated tasking submission to DigitalGlobe

- Automated accounting of product delivery

- Access to live RTMM support

It is important to note that NASA was able to leverage millions of dollars in DOD investments to the original NASA VMOC. Licensing of the DOD VMOC governmentoff-the-shelf(GOTS) technology was agreed upon between GD-AIS and the US Naval Research Lab (NRL). Thus, GOTS code related to the VMOC was used in this project. In return, system development for the NASA demonstration will be incorporated into the NRL core VMOC and will become part of the GOTS code. As a part of this arrangement it was agreed that all NASA-related VMOC code enhancement would be evaluated with regards to impact to the VMOC security accreditation. The DOD accreditation took nearly two years to complete. This is the reason why it was so important to NOT do anything to the NASA VMOC that would perturb the existing accreditation - see Space Asset Selection.

\subsection{Hardware and Software}

As with the previous SAAS demonstration, the VMOC consists of a Mission VMOC utilized for tasking, scheduling, Space Situational Awareness and Information Management. The system consists of a rack-mounted chassis that houses modules interconnected through a standard Internet connection. The computer interfaces to other platforms and equipment via standard connectors and cabling. The physical system resides at Glenn Research Center in Cleveland Ohio and has a connection via the open Internet to field users [Figure 1]. 


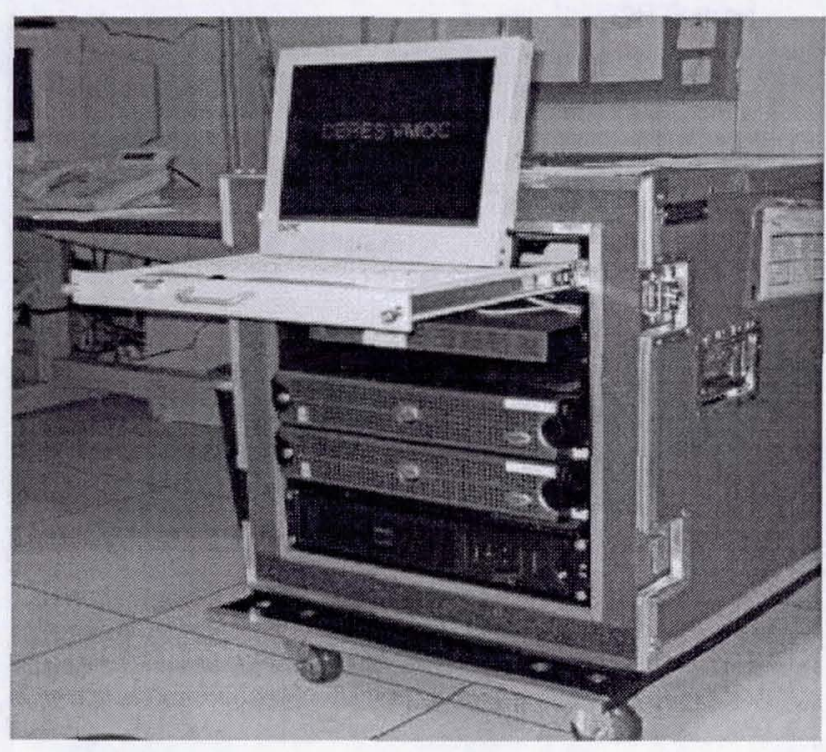

Figure 2 - Great Lakes Operations Center (VMOC at GRC)

The VMOC system utilizes the following commercial-offthe-shelf (COTS) hardware and software:

JBOSS $®$ App server 5.1 Government Addition (GA)

- Adobe ColdFusion ${ }^{\circledR} 9$ Enterprise Edition

- Oracle MySQL 5.1®

- Use of Environmental Systems Research Institute (ESRI) ArcGIS $\otimes$ Map Server over the open internet

- Other VMOC installations use this map server locally, but this was not in the cost scope of this project

- Intel ${ }^{\circledR}$ Multicore-based Server / Windows ${ }^{\circledR} 2008$ Server

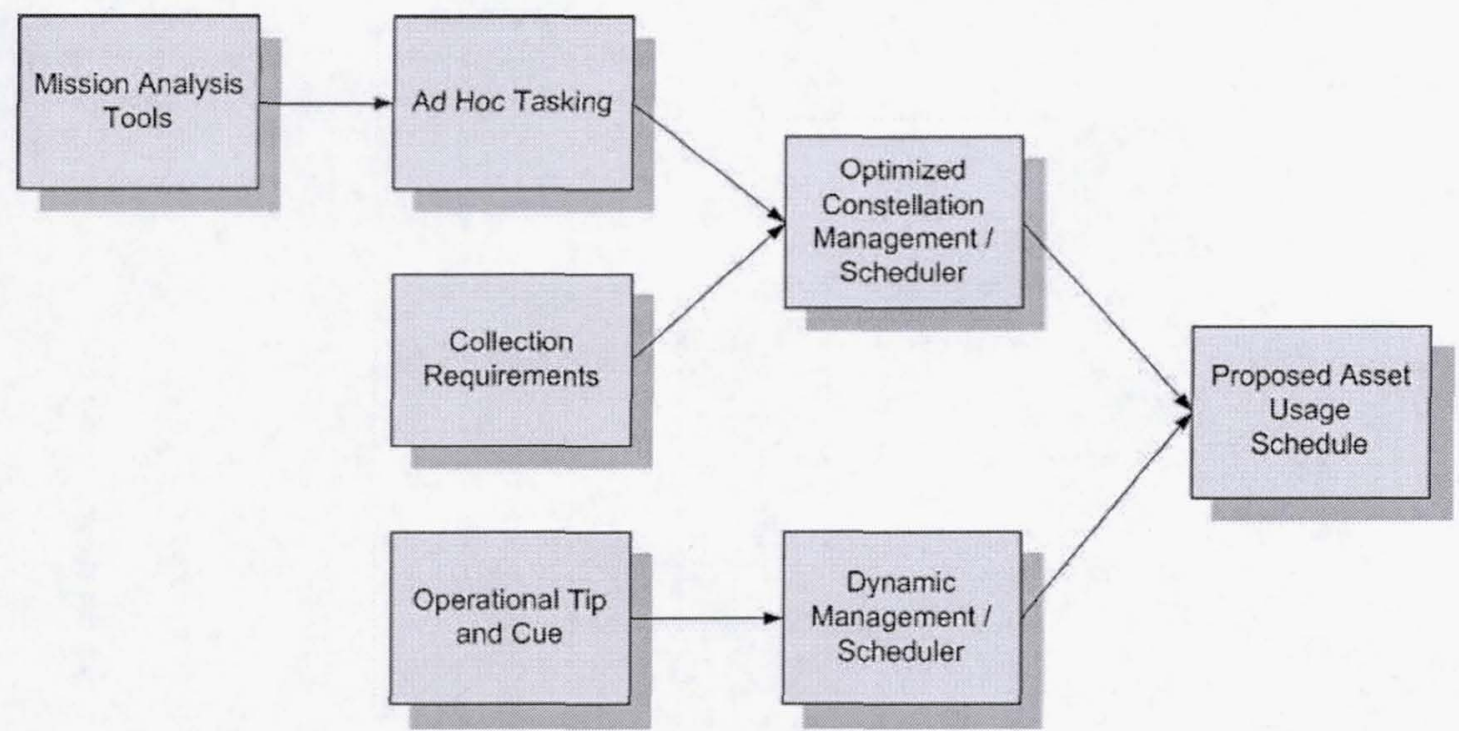

Figure 1 - Mission Planning

\subsection{System Access}

The VMOC uses numerous standard technologies and techniques to manage system access, user privileges and system assets. The VMOC supports the creation and maintenance of user accounts and personal information including definition and assignment of user roles and privileges to control access to individual web pages to specific users. Unauthorized access to pages is detected and access is disabled. The VMOC COTS stack includes robust web sessions management software that custom VMOC software leverages to manage access. Page requests from a user establish a web session where the VMOC can control login and user information. For this demonstration, users authenticate with a username and password. This is considered sufficient for this deployment. More sophisticated techniques such as biometrics or common access cards $(C A C)$ could be deployed. Information is encrypted using account and session level keys. This supports DOD Security Technical Information Guides (STIGS) password selection standards. The session controls page navigation among authorized pages. Direct navigation to pages is supported, but unauthorized pages are blanked.

\subsection{System Flow Process}

The system flow process is illustrated in Figures 2, 4 and 5. Figure 2 shows the mission planning system; Figure 3 shows the accounting system; and Figure 4 shows the external data provider interface.

The mission planning system [Figure 2] is the heart of this VMOC instance. An authorized user, the Emergency Services Coordinator (ESC), will log into the VMOC and request sensor data. Currently, this is done via the Ad Hoc Tasking module by entering the longitude and latitude coordinates of the event of interest and the type of sensor data required and the time requirements for such data. The 
Ad Hoc Tasking module then passes this request on to the Optimized Constellation Management/Scheduler (OCMS). The OCMS runs various algorithms that review which resources are available to meet the required needs. The results are prioritized via rule-base algorithms and passed on to the Proposed Asset Usage Schedule (PAUS) module. The PUAS module is where the results (along with the associated costs of tasking a particular item) are presented to the ESC for validation and execution. This allows the ESC, or other authorized users, to be a gatekeeper prior to committing resources. Execution could be fully automated, but, the current thought is that the gatekeeper function, at least for USGS purposes, is highly desirable, if not actually a requirement in order to control commitments of resources (budgeted dollars).

It is also possible to pre-define collection requirements rather than operate in Ad Hoc mode. The Collection Requirements module allows users to input pre-defined tasking processes and other implementation criteria into VMOC. An example of this would be to command VMOC to "follow a flood" as it progresses from an entry location on a river or flood plain and progresses down stream. The use of predefined tasking processes off-loads the need for continuous monitoring and manual entry from the ESC. As in the ad hoc case, the gatekeeper function can be implemented, requiring authorization prior to execution of tasking during the entire event.

Future systems may also have fully automated "Tip and Cue" capabilities. Automated tip and cue was demonstrated in Phase 1 of the Secure Autonomous Automated Scheduling (SAAS) during late 2009 and early 2010 [2]. Here, USGS earthquake sensor triggers were used as the Tip in order to Cue the United Kingdom Disaster Monitoring Constellation satellite, UKDMC1 to collect satellite imagery.

The Tier One (1) Toolkit [Figure 3] is one of VMOC's mission analysis tools. T1T is a web-based planning tool and guide for commercial, civil, academic, and possibly a limited number of DoD satellite solutions and provides operators with a satellite visualization capability. The Tier One Toolkit (T1T) capabilities monitor on-station space vehicles potentially available to meet urgent and on-going imagery and communication needs. T1T was developed for the Operationally Responsive Space (ORS) Office by the Naval Research Laboratory, intended to provide warfighters and decision makers with information about tasking opportunities for existing space systems. For purposes of the NASA demonstration, the Emergency Service Coordinator and other disaster response officials will be

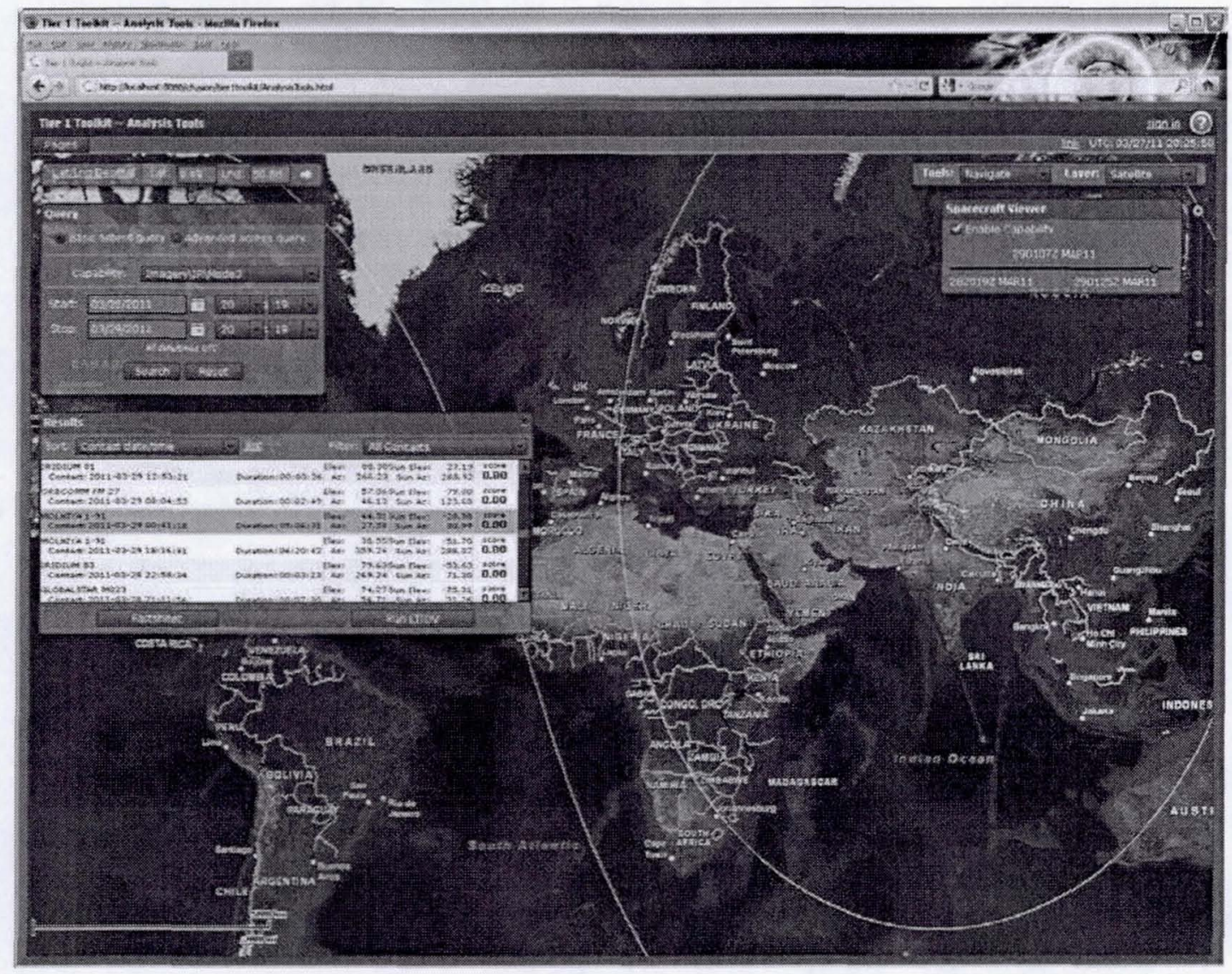

Figure 3 - Tier 1 Toolkit 


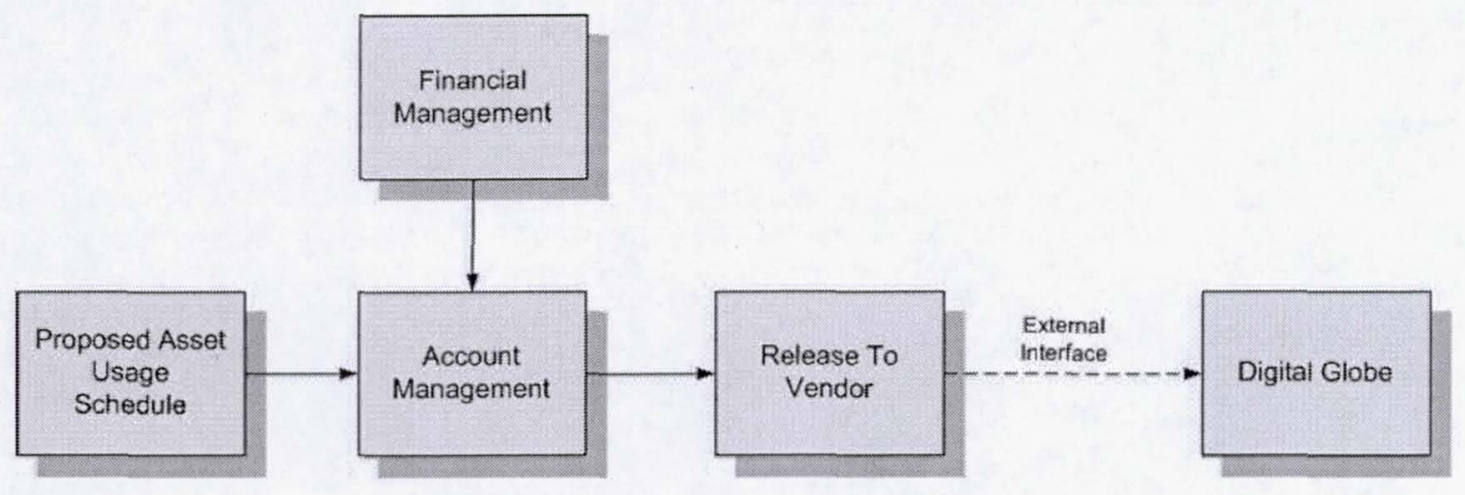

Figure 4 - Accounting System

granted access to the T1T. T1T allows authorized users to search for assets that may meet their needs, and to generate a satellite access request form which can be manually forwarded to the appropriate $\mathrm{DoD}$, commercial, civil and/or approved foreign satellite systems authority for collection. T1T is designed such that non-space savvy users, as well as trained space system operators, can easily search for tasking opportunities.

T1T primarily presents information about non-classified satellite assets in order for users to make informed decisions with regard to potential mission availability and then to request the proper authority to task the asset. T1T does not schedule or task any system or satellite directly. The system is designed to offer unrestricted access to several internal analysis tools, and controlled access to saved-user-data and mission administration functionality. It is important to note that the T1T in the NASA VMOC is on the open Internet and, therefore, directed only at non-classified systems.

Figure 3 shows some of the graphical analysis available to users. Such tools are useful for highly technical users and can be hidden from the novice user who may be only interested in "what sensors are available" and "when they are available".

The Accounting System [Figure 4] receives the proposed tasking opportunities from the Proposed Asset Usage Schedule and presents the associated costs to the ESC. The Accounting Management and Financial Management module work together to ensure there are sufficient financial resources available prior to committing assets as well as keeping track of allocated resources. If sufficient funds are available and the gatekeeper authorizes the commitment, then the request is released to the vendor or other external supplier of sensor data.

Once the gatekeeper releases a request for data products, the external data provider executes that request (or sends notification that the request cannot be executed). When the data order has been executed, the data provider sends notice to the VMOC that the data product is available (or, alternatively, pushes the data product to the VMOC). The system can be set up such that the data provider delivers the satellite data products directly to VMOC or, alternatively, it can send the data directly to a data repository (such as the USGS HDDS) or do both. At any time during the

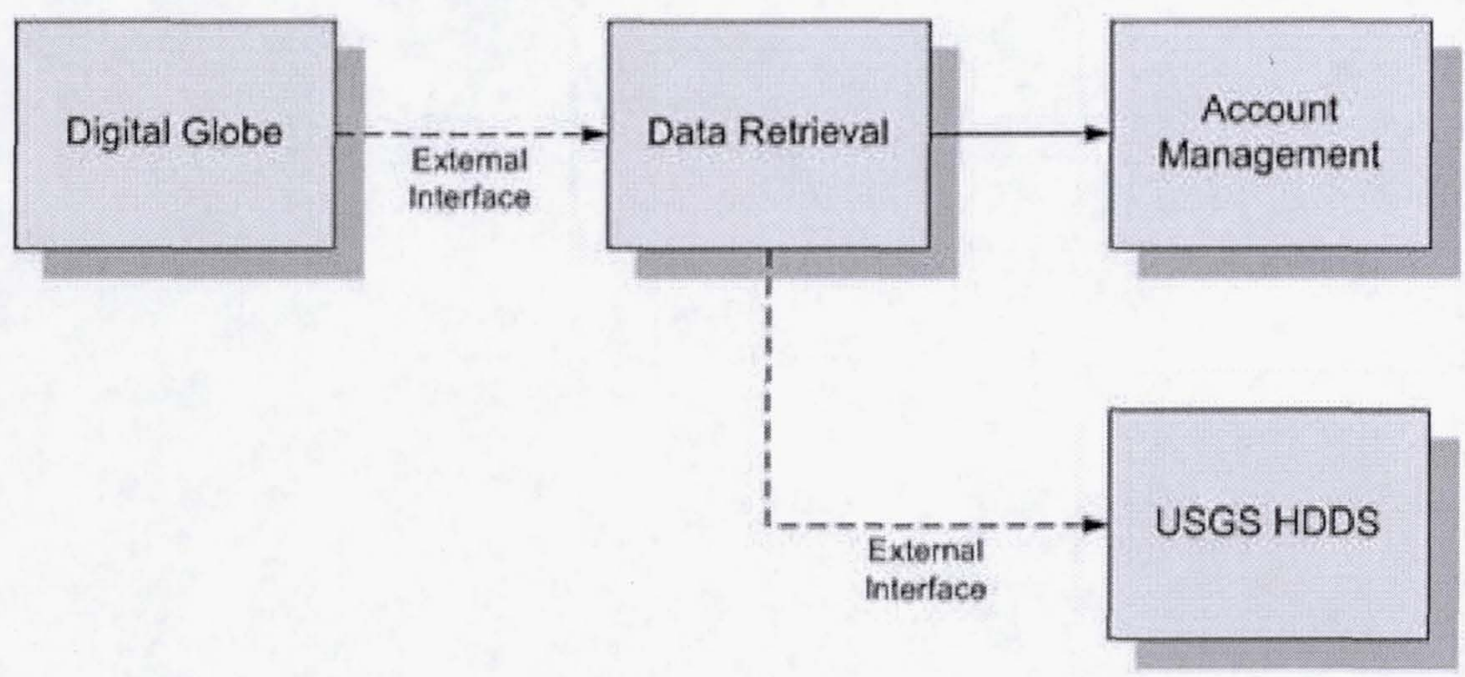

Figure 5 - External Data Provider Interface 
transaction, the ESC can utilize VMOC to monitor the status of request. Once the satellite imagery has been generated and delivered, the VMOC Account Management module records the transaction and debits the appropriate account [Figure 5].

The original thought for data delivery was that since numerous External Data Providers are expected to eventually become a part of the USGS VMOC system, in order to simplify HDDS interfaces, the current implementation requires all data to be sent directly to the VMOC (VMOC then delivers all satellite data to a single, external HDDS data repository). However, upon discussions with USGS it became apparent that USGS already obtains sensor data from the various external providers directly and then massages that data and then makes the USGS standard formatted data product available to the appropriate users [5]. Thus, a decision was made to have the data product retrieval and delivery to the USGS Hazards Data Distribution System (HDDS) directly from DigitalGlobe rather that first passing to the VMOC. This was done for two reasons: 1) to improve time of delivery of vary large data sets; and 2) the interface already exists between HDDS and DigitalGlobe so there is no need to have the VMOC act as a common interface point.

\subsubsection{VMOC / DigitalGlobe Interface}

1. A custom spreadsheet is automatically generated by the VMOC that describes the desired collection.

2. The VMOC transfers the spreadsheet to a DigitalGlobe static web site via File Transfer Protocol (FTP).

3. DigitalGlobe will validate the request and determine whether or not the request can be processed and notify the VMOC.

4. If the request was to be processed, but unforeseen circumstances resulted in cancellation of the task, DigitalGlobe will notify the VMOC so that the VMOC can notify the ESC and take appropriate actions to rectify the situation.

5. Upon successful data collection, DigitalGlobe will notify $\mathrm{VMOC}$ that the data is available.

6. VMOC will then notify USGS that the data product is available and update the Account Management System.

7. USGS HDDS will retrieve the data directly from DigitalGlobe; create the appropriate HDDS data products from the sensor data; and notify users that the HDDS data products are available.

\section{Space Asset Selection}

This particular project had a relatively small budget $(\sim 250 \mathrm{~K})$ and had a very rapid turn around due to lifetimeof-money budget issues - approximately 4 months start to finish. Thus, the space asset had to be readily available and relatively inexpensive.

The first choice was to attempt to use a NASA asset, the EO-1 spacecraft. The EO-1 spacecraft system was particularly attractive since the NASA Goddard Space Flight Center (GSFC) already had an autonomous scheduler for EO-1 that the VMOC could negotiate with [4]. In order to meet cost and schedule, GSFC engineers requested that VMOC interface to their system using available Open Source code that had been integrated into the EO-1 system. Unfortunately, it was determined that use of GSFC's Open Source code in VMOC would require modifications to the existing system security accreditation. Such a modification could not be approved by DOD or the Intelligence Community (IC) without great pains and a year or more of effort. Key to this effort was the desire to leverage DOD and NASA investments in their respective VMOC infrastructure. Therefore, it was imperative to keep both the DOD VMOC and the NASA VMOC security and accreditation compliant. Given the modest budget and tight schedule constraints it was decided that the team needed to pursue other possible satellite systems to be used as the sensor system.

The second choice was to try and use the second-generation United Kingdom Disaster Monitoring Constellation (UKDMC2) platform from Surrey Satellite Technology Limited (SSTL). UK-DMC2 was particularly attractive because NASA GRC and General Dynamics (GD) already had an established relationship with SSTL and GD already had a Technical Assistance Agreement (TAA) with SSTL (which permitted GD to discuss and share regulated technical data with SSTL). To be a part of the current demo, SSTL needed to automate their operational system to allow machine-tomachine requests to SSTL's Mission Planning System and full acknowledgment and acceptance of the request and data return. Unfortunately, negotiations with SSTL determined that the price was prohibitive for this project's budget. However, such support remains highly desirable and will be considered in future VMOC enhancements.

Finally, the team turned to DigitalGlobe for satellite imagery services. DigitalGlobe is a leading global provider of commercial high-resolution earth imagery solutions. In October of 2011, DigitalGlobe was awarded a one-year contract at a funded level of $\$ 37.9$ million by the U.S. Government via the National Geospatial-Intelligence Agency (NGA) under the NGA's new Enhanced Geospatial Intelligence (GEOINT) Delivery (EGD) program. The EGD program, with all options exercised, is expected to procure nearly $\$ 1 \mathrm{~B}$ of commercial imagery products. Interested in fulfilling all of NGA's requirements for a user access portal, DigitalGlobe established a relationship with GD to explore the potential use of GD's VMOC technology. Thus, General Dynamics was able to negotiate an agreement with DigitalGlobe to use DigitalGlobe's space assets for collection and provide a simple machine-to-machine interfaces within the available budget. Note, this DOD commercial imagery interface leverages a multi-year contract with NGA potentially worth over a billion dollars. 


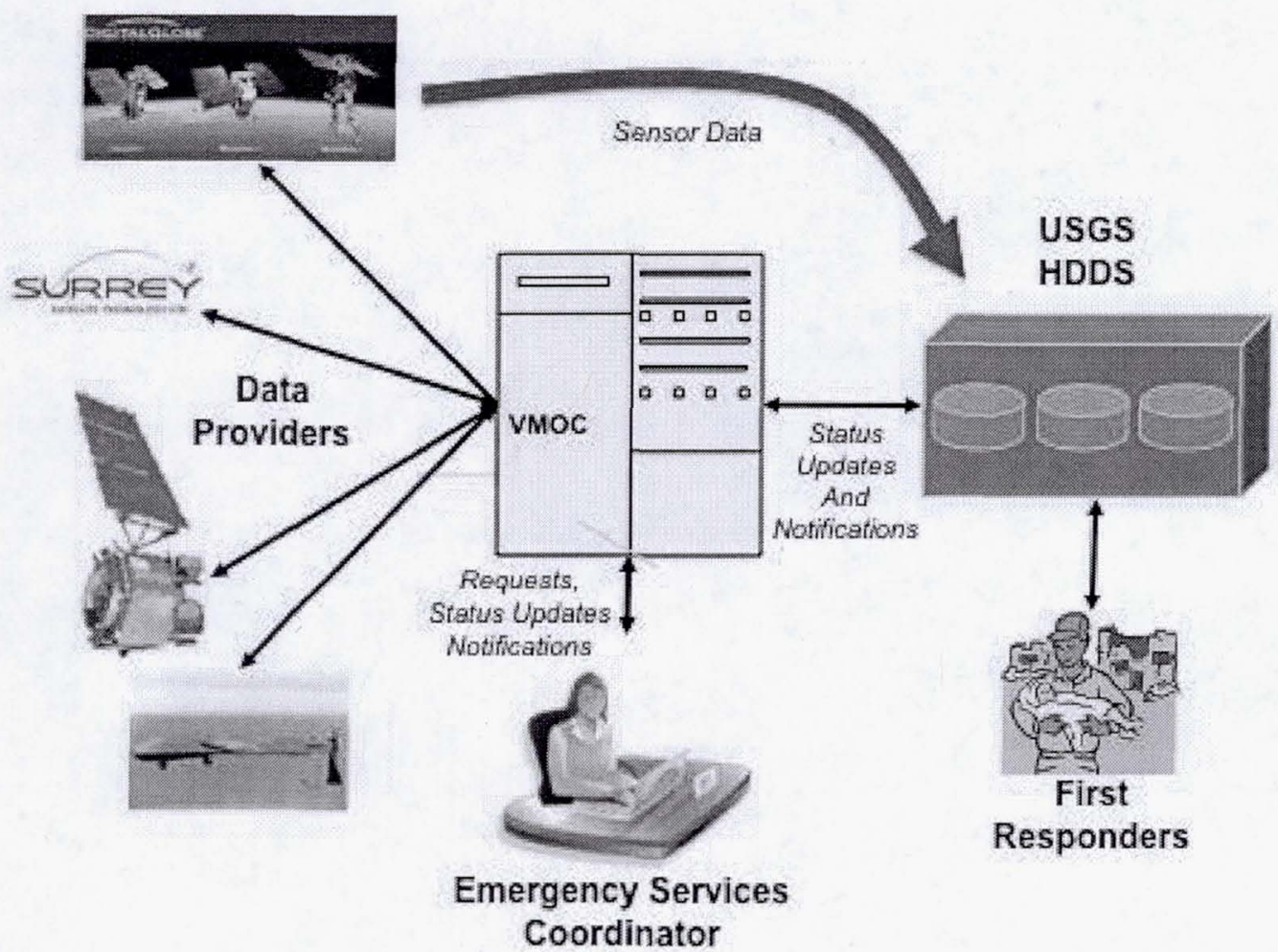

Figure 6-Operations Flow Procedures

\section{Current Concept of Operations}

The current system concept of operations (CONOPS) for this demonstration utilizes all of the major features of VMOC and provides interfaces to the Emergency Services Coordinator, satellite services provider and the U.S. Geological Survey (USGS) Hazards Data Distribution System (HDDS). The HDDS already serves as a data repository that provides quick and easy access to imagery and geospatial data that supports emergency response and recovery operations.

The operations flow procedure is illustrated in Figure 6 and outlined below:

1. Some disaster occurs and the USGS Emergency Services Coordinator (ESC) is notified.

2. The ESC logs into the VMOC and is authenticated as a user.

3. The ESC enters the disaster longitude and latitude, selects the type of sensor data needed, and the time period requirements.

4. The VMOC determines which assets are physically capable of meeting the mission requirements and sends requests to the proper Controlling Entities (CEs) (in this case, DigitalGlobe) to obtain status.

5. The $\mathrm{CE}(\mathrm{s})$ determines the sensor availability and returns a response to the VMOC.
6. The VMOC notifies the ESC of available assets and the expected cost of services.

7. The ESC chooses one, many, or all assets to fulfill the mission need.

8. The VMOC requests a commit of those assets to the $\mathrm{CE}(\mathrm{s})$.

9. The $\mathrm{CE}(\mathrm{s})$ commit their assets (or notes their inability to commit) and notifies the VMOC.

10. The VMOC notifies the ESC which tasks were committed.

11. Once a task has been executed and the data product is available, the $\mathrm{CE}(\mathrm{s})$ notify the VMOC that the request has been executed and data is available.

12. The VMOC notifies the ESC that the task has been executed; informs the HDDS that the data product is available and where to obtain the data; and updates the Accounting Management System.

13. The HDDS obtains the sensor data directly from the data providers, creates the USGS HDDS standard data products, and stores that information on the HDDS.

14. The ESC notifies field uses of the availability of the data and where and how to obtain it. 


\section{Proposed Future Enhancements}

The proposed enhancements to the VMOC are discussed prior to describing the Disaster Relief Operation CONOPS as many of these enhancements will likely be required for an operational system.

\subsection{Digital Rights Management (DRM)}

For our purposes, Digital Rights Management (DRM) are access control technologies and techniques that are used by publishers, copyright holders, and individuals (or organizations) with the intent to limit the use of digital content transaction. DRM is any technology that inhibits uses of digital content that are not desired or intended by the content provider [6]. In the case of data generated in response to disasters, since it is often generated without cost to the disaster responders, it would be highly desirable to have a DRM enabled system to limit access to data products to authorized users only. What is required in VMOC or HDDS for DRM is something akin to an integrated library system (ILS). An ILS is used to track items and has modules for acquisitions (ordering, receiving, and invoicing materials cataloging, and circulation). Some items of interest include:

- Locking digital data to specific computer(s),

- Defining expiration times for digital data,

- Stopping digital data from being shared and distributed across the Internet,

- Defining watermarks for printing and/or viewing, and,

- Restricting printing.

It is important to note that much of the data obtained for disaster management is obtained via bilateral agreements whereby distribution is restricted to particular users and the lifetime of the data may be restricted. Therefore, digital rights management needs to be implemented in the final, operational system. The current operation for HDDS restricts data to particular users via access controls (username and password). However, once that data is downloaded, it could easily be redistributed either accidently or intentionally. Therefore, autonomous data destruction should also be considered to ensure full DRM compliance.

\subsection{Intelligent Collection Requirements}

It is highly desirable to have some intelligence and perhaps even some cognition in the Collection Requirements module whereby the system would be able to predict and follow events without continuous user supervision. For example, in the situation where flooding is occurring, it would be relatively easy to predict the movement of the flood or use additional sensors to monitor the movement of the flood and have the system autonomously request effects. The same could be possible for some weather and wild fire events. Automated "Tip and Cue" processes would also be helpful here.

\subsection{Expert vs. Non-Expert User interface}

The current Disaster Monitoring VMOC is designed to be used by an expert user. It assumes the ESC will have a working knowledge of the available assets (sensors), the type of sensor data required for various disasters, and the data timeliness needed. In the future, it would be extremely beneficial to capture such expertise in the VMOC in such a way that non-experts could readily use the VMOC to the same end. For example, an unsophisticated user may log into the system and indicate that a major earthquake has struck at a particular longitude and latitude. The system would respond back with a prioritized list of sensors that are normally requested for earthquakes and suggested timeliness for various sensor data. Also provided would be the sensor platform and associated costs.

\subsection{Marketing Response}

An additional feature would be to have VMOC suggest to users additional data that may be useful. Similar to the current marketing strategies employed by online shopping services, the VMOC could be designed to suggest services that other past users requested in response to similar circumstances. In other words, a user may request an optical image of a "wild fire" disaster event. VMOC would offer other related services, such as infrared imagery or other types of post-processing.

\section{Disaster Relief Operational CONOPS}

1. A disaster occurs and the USGS Emergency Services Coordinator (ESC) is notified.

2. The ESC logs into the VMOC and is authenticated.

3. The ESC enters the longitude and latitude and selects the type of disaster.

4. The VMOC returns a suggested list of sensor data often required for the particular type of disaster along with a suggested time period.

5. The ESC selects the data product from the list provided.

6. The VMOC determines what assets are available and sends requests to the proper Controlling Entity(ies) (CEs) (in this case, DigitalGlobe).

a. There are cases where the interface between USGS and the requested resource is more or less manual by design or for political reasons. In this case the VMOC will generate the proper paperwork required and fax or email the request to the corresponding authority. In this case auto generating the forms ensures all forms are properly completed.

7. The $\mathrm{CE}(\mathrm{s})$ determines the sensor availability and returns a response to the VMOC.

a. There are many currently available assets where no feedback is provided or where that feedback consists of a phone call or fax. In such instances, the VMOC will have to simply assume that the asset is available, or some form of manual entry will be required. 
8. The VMOC notifies the ESC of available assets and associated costs.

9. The ESC can choose one, many or all assets.

10. The $\mathrm{VMOC}$ requests a commit of those assets to the $\mathrm{CE}(\mathrm{s})$ via whatever means is required for the particular assets (e.g. machine-to-machine, fax, phone, etc...).

11. The $\mathrm{CE}(\mathrm{s})$ commit their assets or notes inability to commit and notifies the VMOC.

12. The VMOC notifies the ESC as to which tasks were committed.

13. The $\mathrm{CE}(\mathrm{s})$ notify the VMOC that the data products are available.

a. At this point, the $\mathrm{VMOC}$ can debit the account and even generate payment.

14. The VMOC notifies the ESC that the data product is available and notifies the HDDS that the data product is available and where the data is located.

15. The HDDS obtains the sensor data directly from the data providers; creates the USGS HDDS standard data products; and stores that information on the HDDS.

16. The ESC notifies field uses of the availability of the data and where and how to obtain it.

In HDDS, field uses can obtain Really Simple Syndication (RSS) notifications by subscribing to the proper services.

\section{NASA's REAL TIME Mission MonitoR}

The NASA Real Time Mission Monitor (RTMM) is a situational awareness tool that integrates satellite, airborne and surface data sets; weather information; model and forecast outputs; and vehicle state data (e.g., aircraft navigation, satellite tracks and instrument field-of-views) for field experiment management [6]. The RTMM is implemented as a service oriented architecture based on community standards and protocols. The RTMM is a client/server-oriented architecture whereby users running client software subscribe to various services (information) from the server side RTMM system via the Internet. The server side RTMM obtains the requested information from both local and remote databases and returns that information to the field users.

The RTMM currently does not task assets. Rather, it takes information from existing databases and redistributes that information in a useful format. The VMOC, on the other hand, does task assets and populates the databases. Thus, the two technologies are complimentary.

For purposes of this task, the original thought was that we could tie the RTMM and VMOC together to task Unmanned Aerial Vehicles (UAVs). Unfortunately, the VMOC does not yet incorporate an active UAV interface (although it does tasking via a tool called "PRISM", which is currently used by the DOD to task military UAVs). In the future, RTMM could be used to provide situational awareness in an emergency response situation while the VMOC could be used to task assets. For this emergency response demonstration, a simple hypertext link to the RTMM is provided to show functionality.

\section{TeChNOlogy REAdiness LeVEL ASSESSMENT}

The overall NASA technology readiness level [8] for the VMOC must address the various aspects of its operations and related software tools.

The VMOC that resides on the SIPRNet is currently tasking both the Tactical Satellite 4 (TacSat-4) and Operational Responsive Space Satellite 1 (ORS-1). TacSat-4 is a UHF Communication satellite for experimental operations. The launch date was September 27, 2011. Transition to operations expected in the spring of 2012. Thus, since this is currently an experimental system, it is at TRL 8. ORS-1 performs visual and infrared imagery for the US Central Command (USCENTCOM). The satellite was launched June 22, 2011 and is fully operational. Therefore, the ORS1 VMOC is at TRL 9. Additional capabilities are in the VMOC pipeline for the near future. They include:

- High Integrity Global Positioning Satellite (GPS) (TRL 7), which uses Iridium signals to augment GPS for anti$\mathrm{jam}$, and is on the path for operational acceptance.

- Tier One Toolkit (TRL 7) which is a suite of spacecraft analysis tools to search over a range of available commercial assets given specific collection criteria and determine what assets are available and when. This system is pending accreditation and is on track to go to the Space and Naval Warfare Systems Command (SPAWAR) as part of the Joint Space Operations Center (JSpOC) Mission System (JMS).

The NASA Secure, Autonomous, Intelligent Controller for Integrating Distributed Emergency Response Satellite Operations system is at TRL 7 as it has been fully demonstrated in a relevant environment. The demonstration of this system to USGS, NGA, DOD TENCAP, and others has provided valuable insight into the security, accounting, and user feedback interfaces that need further work before the system can become fully operational. 


\section{REFERENCES}

[1] William D. Ivancic, Dave Stewart, Jon Walke, Larry Dikeman, Steven Sage, Eric Miller, James Northam, Chris Jackson, John Taylor, Scott Lynch, Jay Heberle, Lloyd Wood, Phillip Paulsen: "Virtual Mission Operations of Remote Sensors with Rapid Access To and From Space," AIAA-2010-2305, Huntsville, Alabama, SpaceOps 2010, April 26-30, 2010

[2] Jon G. Walke, Larry Dikeman, Stephen P. Sage, and Eric M. Miller General Dynamics Advanced Information Systems, Vandenberg Air Force Base, California, "Secure Autonomous Automated Scheduling (SAAS) Phase 1 Final Report" NASA Contractor Report NASA/CR.2010216097, March 2010

[3] William Ivancic, Phil Paulsen, Dave Stewart, Dan Shell, Lloyd Wood, Chris Jackson, Dave Hodgson, James Northam, Neville Bean, Eric Miller, Mark Graves and Lance Kurisaki: "Secure, Network-Centric Operations of a Space-Based Asset: Cisco Router in Low-Earth Orbit (CLEO) and Virtual Mission Operations Center (VMOC),"NASA/TM-2005-213556, May, 2005

[4] Daniel Mandle, Pat Cappelaere, Stuart Frye, Rob Sohlberg, Lawrence Ong, Steve Chien, Daniel Tran, Ashley Davies6, Don Sullivan7, Stefan Falke8, Steve Kolitz, Peishing Zhao, Liping Di, Nengchen Chen, Geyong Yu, David Smithbauer, Stephen Ungar, Linda Derezinski, Mike Botts" Sensor Web 2.0: Connecting Earth's Sensors via the Internet," NASA Earth Science Technology Office (ESTO) Conference, ]une 24-26, 2008

[5] https://hdds.usgs.gov/hdds/, March 2012

[6] http://en.wikipedia.org/wiki/Digital_rights_management, February 2012

[7] http://rtmm.nsstc.nasa.gov/, February 2012

[8] Chris Moore: "TRL Usage at NASA," DoDCost Analysis Symposium, February 20, 2008, http://209.48.244.135/DODCAS\%20Archives/41st\%20D ODCAS $\% 20(2008) /$ Track $\% 203 \% 20$ $\% 20$ Acquisition\%20and\%20Logistics/T3S1b_Moore.pdf, March 2012

\section{APPENDIX A - ACRONYMS}

AIST - Advanced Information Systems Technology ATI - Advanced Technology Insertion (under ESTO) CEOS - Committee on Earth Observing Satellites DOD United States Department of Defense

CE - Controlling Entity CONOPS - Concept of Operations COTS - Commercial-off-the-shelf DMC - Disaster Monitoring Constellation

DRM - Digital Rights Management EGD-Enhanced GEOINT Delivery EROS - Earth Resources Observation and Science

ESC - Emergency Service Coordinator

ESTO - Earth Science Technology Office

FTP - File Transfer Protocol

GD-AIS - General Dynamics Advanced Information

Systems

GEOINT - Geospatial-Intelligence

GOTS - Government-off-the-shelf

GPS - Global Positioning Satellite

GRC - NASA Glenn Research Center

GSFC - NASA Goddard Space Flight Center

HDDS - Hazards Data Distribution System

HTTP - Hypertext Transfer Protocol

ILS - Integrated Library System

JSC - NASA Johnson Space Center

JSpOC - Joint Space Operations Center

NGA - National Geospatial-Intelligence Agency

NRL - Naval Research Laboratory

OCMS - Optimized Constellation Management/Scheduler RTMM - Real Time Mission Monitor ORS - Operationally Responsive Space ORS-1 - Operational Responsive Space satellite \#1 SAAS - Secure, Autonomous, Automated Scheduling SIPRNET - Secure Internet Protocol Router Network SOCOM - Space Operations Command SPAWAR - Space and Naval Warfare Systems Command SSTL- Surrey Satellite Technology Limited

STIGS-DOD Security Technical Information Guides

TacSat-4 - Tactical Satellite \#4

T1T - Tier One (1) Toolkit

TAA- Technical Assistance Agreement

TDRSS - Tracking and Data Relay Satellite System

TENCAP - United States Air Force Tactical Exploration of

National Capabilities

TRL - Technology Readiness Level

UAV - Unmanned Aeronautical Vehicle

UKDMC-1 - Version 1 of the United Kingdom Disaster

Monitoring Constellation satellite by SSTL

UKDMC-2 - Version 2 of the United Kingdom Disaster Monitoring Constellation satellite by SSTL

USCENTCOM - United States Central Command

USGS - United Stated Geological Service

USN - Universal Space Network

VMOC - Virtual Mission Operations Center

WGISS - Working Group on Information Systems and

Services, supporting the Committee on Earth Observing Satellites 